\title{
The reliability of and the relation between non-symbolic numerical distance effects in comparison, same-different judgments and priming
}

\author{
Delphine Sasanguie ${ }^{\mathrm{a}, *}$, Emmy Defever $^{\mathrm{a}}$, Eva Van den Bussche ${ }^{\mathrm{b}}$, Bert Reynvoet ${ }^{\mathrm{a}}$ \\ a Department of Psychology, University of Leuven, Belgium \\ ${ }^{\mathrm{b}}$ Department of Psychology, University of Brussels, Belgium
}

\section{A R T I C L E I N F O}

Article history:

Received 28 June 2010

Received in revised form 31 August 2010

Accepted 12 October 2010

Available online $\mathrm{xxxx}$

\section{PsycINFO classification code:}

2340

\section{Keywords:}

Number processing

Comparison

Same-different judgment

Priming

Reliability

Non-symbolic stimuli

\begin{abstract}
A B S T R A C T
The development of number processing is generally studied by examining the performance on basic number tasks (comparison task, same-different judgment, and priming task). Using these tasks, so-called numerical distance effects are obtained. All these effects are generally explained by assuming a magnitude representation related to a mental number line: magnitudes are represented from left to right with partially overlapping representations for nearby numbers. In this study, we compared the performance of adults on these different tasks using non-symbolic stimuli. First, we investigated whether the effects obtained in these behavioral tasks are reliable. Second, we examined the relation between the three different effects. The results showed that the observed effects in the case of the comparison task and the same-different task proved to be reliable. The numerical distance effect obtained in the priming task, however, was not reliable. In addition, a correlation was found between the distance effects in the comparison task and the same-different task. The priming distance effect did not correlate with the other two effects. These results suggest important differences between distance effects obtained under automatic and intentional task instructions regarding the use of them as indices of mathematical ability.
\end{abstract}

(c) 2010 Elsevier B.V. All rights reserved.

\section{Introduction}

How people understand and process numerical quantities has been intensively studied by examining the performance on three basic number tasks: comparison, same-different judgment and priming (for reviews, see Ansari, 2008; Cohen-Kadosh, Lammertyn, \& Izard, 2008). The effects obtained with these tasks provide an insight on how magnitude is represented in the human brain. In all three tasks, a so-called numerical distance effect is observed: in comparison and same-different judgments, reaction times increase with decreasing distance between the two presented numerosities. In a priming task, the reaction times decrease if the numerical distance between two sequentially presented numerosities is small. Traditionally, all of these effects are explained by assuming a magnitude representation related to a mental number line (Dehaene, 1997). On this mental number line, magnitudes are represented on a left-to-right oriented line as a distribution around the true location of each specific number, with partially overlapping representations of other nearby numbers. This implies that, whenever a magnitude is presented, not only the representation of that specific magnitude will be activated, but also

\footnotetext{
* Corresponding author. Subfaculty of Psychology and Educational Sciences, Department of Psychology, K.U.Leuven - Campus Kortrijk, Etienne Sabbelaan 53; 8500 Kortrijk, Belgium. Tel.: +3256246037.

E-mail address: Delphine.Sasanguie@kuleuven-kortrijk.be (D. Sasanguie).
}

partially the representation of the nearby numbers. This organization of the magnitude representation can explain each of the numerical distance effects in a specific way.

The effect generated by the comparison task, is the comparison distance effect (CDE) (e.g. Moyer \& Landauer, 1967). When subjects have to decide which of two magnitudes is numerically larger, they will be slower in discriminating between two numbers that are numerically close to each other. For instance, subjects make more errors and need more time when comparing the pair "8-9" than the pair "1-9". Originally, Restle (1970) proposed that the CDE was caused by overlapping representations for magnitudes: nearby magnitudes overlap more, making it more difficult to discriminate between them. This explanation for the CDE was later incorporated in more recent models of numerical cognition (e.g. Dehaene, Piazza, Pinel, \& Cohen, 2003). The CDE has already proven to be a robust phenomenon. It emerges for numbers presented in various formats (e.g. Buckley \& Gillman, 1974) and it seems especially reliable in the case of nonsymbolic quantities (Maloney, Risko, Preston, Ansari, \& Fugelsang, 2010). Sekuler and Mierkiewicz (1977) demonstrated that the effect is also observed in five-year-old kindergarteners and that the size of the CDE decreases with increasing age (see also Holloway \& Ansari, 2009).

In the same-different task, subjects have to decide whether two magnitudes are numerically equal (same) or not (different). Again, a distance effect, which we will refer to as the same-different distance effect or SDDE, is observed: the reaction time needed for a 'different' 
decision is moderated by the numerical distance between the two magnitudes (e.g. Dehaene \& Akhavein, 1995). For example, deciding that "SEVEN-8" have different numerical values takes longer than doing this for "ONE-8". It is assumed that this effect is also originated on the mental number line: two numbers that are far apart have less representational overlap, making it, in a later stage of processing, easier to discriminate between them. In contrast, if the numerical distance is small and consequently the representational overlap substantial, it is harder to distinguish between both. In contrast with the CDE, Duncan and McFarland (1980) found that the SDDE does not interact with age: the effect is equally strong in kindergarteners, $1 \mathrm{st}$, 3rd and 5th graders and adults.

Finally, also in priming studies a distance effect is observed (i.e. the priming distance effect or PDE). This effect is manifested by faster responses to targets when they are preceded by a numerical close prime. For instance, the digit " 4 " is named faster when it is preceded by "3" than by "2" (e.g. Reynvoet, Brysbaert, \& Fias, 2002). In terms of the mental number line explanation, it is assumed that a prime number not only activates its own magnitude representation, but also that of nearby numbers. When the prime is followed by a numerically close target, reaction times will be faster, because the preceding prime already partially activated the target representation. The PDE is also present in children and is similar in younger and older children and adults (Reynvoet, De Smedt, \& Van den Bussche, 2009).

Although the three numerical distance effects are thought to reflect the same processing mechanism (i.e. representational overlap), it should be noted from the previous section that developmental work points towards an unexpected dissociation between the different numerical distance effects. Whereas several studies have observed that the CDE decreases with increasing age (e.g. Holloway \& Ansari, 2009; Sekuler \& Mierkiewicz, 1977), this interaction with age does not seem to appear for the SDDE (Duncan \& McFarland, 1980) and the PDE (Reynvoet et al., 2009). The decreasing CDE is explained by assuming that the mental number line becomes increasingly more precise as schooling advances. There is, in other words, a developmental shift, due to increasing precision of the magnitude representations with increasing age (Noel, Rouselle, \& Mussolin, 2005; Sekuler \& Mierkiewicz, 1977). However, such an explanation is difficult to reconcile with the assumption that all three distance effects stem from the same underlying magnitude representation: the developmental changes should be observed for all effects, independent of the task. In addition, Van Opstal, Gevers, De Moor, and Verguts (2008) also found inconsistencies between numerical distance effects in studies with adults. In their study, they investigated the CDE and the PDE in letters and numbers. Whereas both effects were, as expected, observed in the number condition, only a CDE (and no PDE) was present in the letter condition. Again, this shows that the idea of an identical origin of both effects is debatable.

One solution to account for these inconsistencies between numerical distance effects may be provided by neural network models (Dehaene \& Changeux, 1993; Van Opstal \& Verguts, 2010; Verguts, Fias, \& Stevens, 2005). Whereas the CDE was originally explained by overlapping representations for different magnitudes, Verguts et al. (2005) showed that representational overlap is not a necessary condition for a CDE to emerge. They demonstrated that the CDE can be alternatively explained by the differential strength of connections between the magnitude nodes on the number line and the response nodes ("smaller" and "larger"). For example, when comparing magnitudes with a fixed standard (e.g. 5), the connection weights between the magnitude node and the output node "larger than the standard" will increase linearly with increasing magnitude. Similarly, the connection weights between the magnitude node and the output node "smaller than the standard" will decrease with increasing magnitude. These connection weights evoke the CDE: the activation of the output node "larger than the standard" or "smaller than the standard" increases when the numerical distance between the magnitude and the fixed standard number increases, which results in faster latencies. The observations by Holloway and Ansari (2008) that a developmental change in the $\mathrm{CDE}$ is observed in numerical and nonnumerical magnitude judgments (e.g. luminance judgments) are in line with the idea that the CDE possibly reflects a general decision process. In contrast, the SDDE (Dehaene \& Changeux, 1993; Van Opstal \& Verguts, 2010) and the PDE (Van Opstal et al., 2008; Verguts et al., 2005) can only be simulated by assuming representational overlap. On the basis of these computational models, dissociations between the $\mathrm{CDE}$ on the one hand, and the SDDE and the PDE on the other hand can be expected as they address different mechanisms, i.e. decisional mechanisms and representational overlap respectively.

In addition to computational modeling, Cohen-Kadosh and Walsh (2009) recently also proposed that dissociations between numerical distance effects might be caused by either automatic or intentional numerical processing. In their proposal, the authors assume that the automatic activation of the magnitude representation is initially crude, but can be further refined through intentional processing. This distinction is crucial for our numerical distance effects under consideration as the CDE and the SDDE are the result of intentional processing (i.e. when the numerical difference is essential for the task requirements), whereas the PDE is considered as the result of automatic numerical activation (Reynvoet \& Notebaert, 2009). Studies examining the PDE have adopted a wide range of different priming paradigms. In the present study, we used a variant in which a response, i.e. smaller or larger than 5 , on both prime and target is needed (see also CohenKadosh, 2008, Herrera \& Macizo, 2008; Koechlin, Naccache, Block, \& Dehaene, 1999; Reynvoet et al., 2009), resulting in a large PDE. However, although the numerical comparison of both prime and target with the standard 5 can be considered as intentional, the way in which the magnitude of the prime affects the reaction time on the target, can be considered as an automatic process if a short response-to-stimulusinterval is used (see also Cohen-Kadosh, 2008). Based on this, a difference between the CDE and the SDDE on the one hand and the PDE on the other hand might be expected.

In order to shed further light on the potential dissociations between the different numerical distance effects (i.e. CDE, SDDE and PDE) the present study aims to investigate the relationship between them obtained in a comparison, a same-different and a priming task, within the same subject sample. Until now, most studies have explicitly focused on one of these effects making a direct comparison between them impossible. However, first we will examine the reliability of the numerical distance effects, because the main reason why a zero correlation between effects is sometimes found, is that the individual effects themselves are not stable and reliable. Several recent studies have looked into the relation between numerical distance effects and measures of mathematical ability (e.g. De Smedt, Verschaffel \& Ghesquière, 2009; Holloway \& Ansari, 2009; Iuculano, Tang, Hall, \& Butterworth, 2008; Mundy \& Gilmore, 2009; Soltész, Szücs, \& Szücs, 2010). In these studies, it is not uncommon that only a few significant correlations are found, which might be due to a lack of reliability.

In a first experiment we describe the results of a comparison task. In a second experiment we report the results of a same-different judgment. The third experiment consists of a priming task. Finally, the reliability of the distance effects obtained in these experimental tasks is examined and the correlations between the numerical distance effects are computed. In this study, the numerical distance effects were examined using the same non-symbolic stimuli in all three experiments. When symbolic stimuli (e.g. digits) are used as stimuli, Cohen (2009) showed that subjects rely on physical similarity to make a same-different decision making the effects obtained in the three tasks incomparable. ${ }^{1}$

\footnotetext{
${ }^{1}$ In our study, we replicated the findings of Cohen (2009). When subjects had to conduct a same-different decision on two digits, they relied on physical similarity. Consequently, no distance effect was found in this case making a comparison of the numerical distance effects across tasks impossible.
} 


\section{Experiment 1: comparison task}

\subsection{Method}

\subsubsection{Participants}

Fifty students participated for a small reward. All the participants took part in a comparison task, a same-different task and a priming task with symbolic and non-symbolic stimuli. As mentioned, we focus on the data from the experiments with the non-symbolic stimuli, for the sake of the comparability. One subject was excluded from all experiments because she was diagnosed with dyslexia and dyscalculia. Furthermore, in the comparison experiment with non-symbolic stimuli, two subjects were excluded from the data: one because he was substantially slower $(>3 S D)$ than the other participants, and the other because she made more errors $(>3 S D)$ than the other subjects. Consequently, the final sample for this first experiment consisted of 47 participants (mean age $=19.32$ years; $S D=5.78 ; 19$ males, 28 females).

\subsubsection{Apparatus}

The task was conducted using a 15-inch color screen connected to a computer running the Windows XP operating system. Stimulus presentation and the recording of behavioral data (reaction time and error rates) were controlled by E-prime 1.1 (Psychology Software Tools, http://www.pstnet.com).

\subsubsection{Stimuli}

Two white-filled circles each containing a set of black dots were simultaneously presented on a black background. One circle was situated $4.25 \mathrm{~cm}$ left and the other $4.25 \mathrm{~cm}$ right from a fixation cross in the center of the screen. The radius of the circles was $3.5 \mathrm{~cm}$. The dot patterns consisting of 1 to 9 dots were generated using MatLab software (Dehaene et al., 2005). Dot size and total area were systematically varied in order to prevent the consistent use of perceptual features to compare the dot arrays. Each possible combination of quantities was presented (except for equal quantities), resulting in $9 \times 8=72$ trials per block.

\subsubsection{Procedure}

A fixation cross was first presented on each trial. The fixation cross disappeared after $600 \mathrm{~ms}$ and was replaced by two stimuli (i.e. the two white circles each containing a dot pattern). The stimuli remained on the screen until a button was pressed. The intertrial interval was $1000 \mathrm{~ms}$. Prior to the experiment, verbal instructions were presented on the screen, indicating that pairs of dot patterns consisting of 1 to 9 dots would be presented. The subjects were asked to decide as quickly and as accurately as possible which circle contained the most dots. They were instructed to press a left or right button ('a' or 'p' on an AZERTY keyboard) corresponding to the side where more dots were presented. First, the subjects were presented with 10 training trials, randomly selected from the list. Then, two blocks of 72 experimental trials followed, separated by a short break. Participants were tested in small groups of about 12 . The experiment took approximately $15 \mathrm{~min}$.

\subsection{Results}

\subsubsection{Reaction times}

In Table 1, mean reaction times (RT) and mean error rates for the eight distances ( $\mathrm{d} 1-\mathrm{d} 8$ ) of the comparison task in block 1 and block 2 are presented. A repeated measures analysis was conducted on the median reaction times of participants' correct responses (on average $2.25 \%$ of the trials were errors), with distance (eight levels: distances 1-8) and block (two levels: experimental blocks 1 and 2) as withinsubject factors. A significant main effect of distance was found (see Table 1): subjects responded on average $175 \mathrm{~ms}$ slower on distance 1 in comparison to distance $8(F(7,40)=19.35, p<.001)$. We also observed a main effect of block, characterized by faster reaction times (on average $33 \mathrm{~ms}$ faster) in the second block $(F(1,46)=26.29$, $p<.001)$. In addition, a significant block $\times$ distance interaction was found: $F(7,40)=2.39, p=.039$. Reaction times were significantly faster on block 2 for distances 1 to 5 (all $t(46)>3.83$, all $p<.01$ ), while there was no difference anymore between both blocks from distance 6 onwards.

\subsubsection{Error rates}

Similar to the reaction time data, a repeated measures analysis was conducted for the error rates, again using distance and block as withinsubject factors. A main effect of distance was found $(F(7,40)=15.69$, $p<.001$ ): subjects made significantly more errors (on average $13 \%$ ) on distance 1 in comparison to distance 8 (see Table 1). The main effect of block and the interaction were not significant $(F<1$ and $F(7,40)=1.42$, $p=.22$ respectively).

\section{Experiment 2: same-different judgment}

\subsection{Method}

\subsubsection{Participants}

The participants were the same as in Experiment 1. In the samedifferent task, one subject who made more errors $(>3 S D)$ than the other participants was excluded. Thus, in total, the final sample for this experiment consisted of 48 subjects (mean age $=19.33$ years, $S D=5.71 ; 19$ males, 29 females).

\subsubsection{Apparatus and stimuli}

The apparatus and the stimuli were the same as those in Experiment 1 , except that nine 'same' trials (i.e. trials in which the two white stimulus-circles contained an equal number of dots) were added which were presented 4 times per block, leading to 36 same and 72 different trials. Two blocks of these 108 experimental trials were presented.

The same trials were not further analyzed because the SDDE only manifests itself in the different trials. Again, in this experiment, dot size and total area of the trials was systematically varied in order to prevent the consistent use of perceptual features to compare the dot arrays.

\subsubsection{Procedure}

The procedure was highly similar as in experiment 1 , only the instructions differed: here the subjects had to decide whether the number of dots of two simultaneously presented dot patterns was the same or different and consequently press the corresponding button (' $a$ ' for same and ' $p$ ' for different on an AZERTY keyboard). To avoid noise and confusion on behalf of the participants and to increase the similarity with the other experiments, the response assignment was not varied. The experiment took approximately $15 \mathrm{~min}$.

Table 1

Mean reaction times (RT), mean error rates and corresponding (standard deviations) for the eight distances (d1-d8) of the comparison task in block 1 and block 2 .

\begin{tabular}{|c|c|c|c|c|c|c|c|c|c|}
\hline & & d1 & $\mathrm{d} 2$ & d3 & $\mathrm{d} 4$ & d5 & d6 & d7 & d8 \\
\hline \multirow[t]{2}{*}{ RT (ms) } & Block 1 & $663.8(167.8)$ & $574.7(129.3)$ & 528.8 (95.0) & 502.5 (92.3) & $486.5(87.0)$ & $465.0(70.6)$ & $468.0(94.6)$ & $454.1(67.4)$ \\
\hline & Block 2 & $589.5(138.5)$ & $528.2(103.2)$ & $492.8(80.4)$ & 471.3 (74.9) & 449.1 (66.8) & $448.9(79.2)$ & 449.4 (66.6) & $448.6(91.3)$ \\
\hline \multirow[t]{2}{*}{ Error rates (\%) } & Block 1 & $.14(.11)$ & $.04(.06)$ & $.01(.02)$ & $.02(.04)$ & $.01(.04)$ & $.01(.03)$ & $.00(.00)$ & $.00(.00)$ \\
\hline & Block 2 & $.12(.09)$ & $.05(.06)$ & $.02(.03)$ & $.01(.03)$ & $.02(.04)$ & $.01(.02)$ & $.02(.06)$ & $.00(.00)$ \\
\hline
\end{tabular}


Table 2

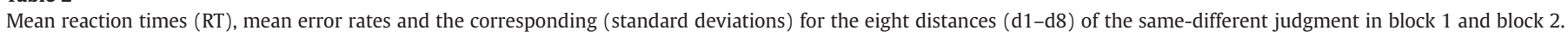

\begin{tabular}{|c|c|c|c|c|c|c|c|c|c|}
\hline & & $\mathrm{d} 1$ & $\mathrm{~d} 2$ & d3 & $\mathrm{d} 4$ & $\mathrm{~d} 5$ & d6 & $\mathrm{d} 7$ & $\mathrm{~d} 8$ \\
\hline \multirow[t]{2}{*}{$\mathrm{RT}(\mathrm{ms})$} & Block 1 & $988.7(373.0)$ & $818.3(218.5)$ & 777.3 (185.9) & $717.0(192.4)$ & $671.0(138.1)$ & $667.1(173.5)$ & $660.8(158.2)$ & $646.0(202.8)$ \\
\hline & Block 2 & $927.7(341.4)$ & 806.3 (206.9) & $730.2(154.2)$ & $711.8(168.0)$ & 641.9 (133.9) & $624.9(123.4)$ & $611.6(133.1)$ & $693.7(288.4)$ \\
\hline \multirow[t]{2}{*}{ Error rates (\%) } & Block 1 & $.25(.16)$ & $.11(.11)$ & $.05(.08)$ & $.03(.06)$ & $.03(.08)$ & $.04(.09)$ & $.05(.12)$ & $.03(.12)$ \\
\hline & Block 2 & $.21(.14)$ & $.07(.07)$ & $.04(.06)$ & $.03(.06)$ & $.03(.06)$ & $.04(.09)$ & $.02(.07)$ & $.02(.10)$ \\
\hline
\end{tabular}

\subsection{Results}

\subsubsection{Reaction times}

Similar as in Experiment 1, a repeated measures analysis was conducted on the median reaction times of the participants' correct answers on different trials, using distance and block as within-subject factors. Table 2 provides an overview of the mean reaction times (RT) and the mean error rates for the eight distances (d1-d8) of the samedifferent judgment in block 1 and block 2. Errors (on average $8.0 \%$ ) were excluded from the analysis. A significant main effect of distance was found $(F(7,41)=13.79, p<.001)$, with reaction times on average $288 \mathrm{~ms}$ slower on distance 1 in comparison to distance 8 . In addition, a significant main effect of block was also present $(F(1,47)=5.24$, $p=.03)$ : subjects were on average $25 \mathrm{~ms}$ faster in the second block. An interaction effect of block by distance was absent $(F(7,41)=1.03$, $p=.42$ ).

\subsubsection{Error rates}

A repeated measures analysis was conducted for the error rates on the different trials, using distance and block as within-subject factors. A main effect of distance was found $(F(7,41)=14.91, p<.001)$, indicating that subjects made significantly more errors (on average $21 \%$ ) on distance 1 in comparison to distance 8 (see Table 2 ). A significant main effect of block was also observed $(F(1,47)=10.84, p<.01)$; subjects made 3\% less errors in the second block than in the first block. An interaction effect of distance by block was lacking in this error rate analysis $(F(7,41)=1.53, p=.18)$.

\section{Experiment 3: priming task}

\subsection{Method}

\subsubsection{Participants}

Again, the same sample participated in this third experiment. In this experiment, three subjects were excluded from the sample of 49 subjects: one person was slower $(>3 S D)$ than the others, another person made more errors $(>3 S D)$, and yet another person was $2 S D$ faster than the group mean. Inspection of the data of this last subject showed that the response on the target had been anticipated on the basis of the prime. Therefore, this subject was also excluded from the analyses. Thus, the final sample of this experiment, was 46 subjects (mean age $=19.35$ years, $S D=5.84 ; 18$ males, 28 females).

\subsubsection{Apparatus and stimuli}

The apparatus and the stimuli were the same as those in Experiments 1 and 2. In this task, the prime stimuli were dot patterns consisting of 1 to 9 dots, excluding 5 . Targets were limited to dot patterns with numerosities $1,4,6$ and 9 , resulting in 8 (prime) $\times 4$ (target) $=32$ possible prime-target combinations from distance 1 (e.g. prime 2-target 1) to distance 3 (e.g. prime 6-target 9). After ten training trials (selected at random from the list), two blocks of experimental trials were presented. This set of trials contained congruent and incongruent trials: on a congruent trial, prime and target evoke the same response (for instance prime 2 followed by target 4: both evoke the same response 'smaller than five'); on an incongruent trial, prime and target evoke different responses (e.g. prime 2-target 6; the first is 'smaller than five', the second 'larger than five'). Response congruent trials were presented twice in each block, leading to
48 (16 incongruent and 32 congruent) trials per block. Because there were twice as many congruent trials than incongruent trials, subjects could use crude aspects of the magnitude relation between prime and target to some extent, i.e. the likelihood that both prime and target are on the same side of the standard (e.g. both smaller than five) and thus require the same response is above chance. However, because the PDE will be calculated solely on the basis of the congruent trials, these crude aspects of the magnitude relation are not sufficient to influence the PDE in an intentional way, since the PDE is based on more refined characteristics of the magnitude relation between prime and target.

\subsubsection{Procedure}

The fixation cross disappeared after $600 \mathrm{~ms}$ and was followed by a prime that remained in the center of the screen until a response (i.e. larger or smaller than five) was detected. Then a blank screen was shown for $200 \mathrm{~ms}$, followed by the presentation of the target. The target also remained in the center of the screen until a response was detected. After the response on the target, a blank screen was shown for $2000 \mathrm{~ms}$, serving as the intertrial interval. Participants were asked to decide as quickly and as accurately as possible whether the number of dots presented on the screen was smaller or larger than five by pressing a left button ('a' on an AZERTY keyboard) for dot patterns smaller than five and a right button ('p') for dot patterns larger than five. All participants used the same response assignment, because of its consistency with the association between space and magnitude (i.e. the SNARC-effect; Dehaene, Bossini, \& Giraux, 1993). None of the participants was told that the numerical relation between both numbers was of importance for this experiment. This experiment took approximately $15 \mathrm{~min}$.

\subsection{Results}

In line with previous studies (e.g. Koechlin et al., 1999; Reynvoet et al., 2009), the PDE was computed solely on the basis of response congruent trials. Response incongruent trials lead to a response interference effect that masks the numerical distance effect (e.g. Reynvoet, Caessens, \& Brysbaert, 2002).

\subsubsection{Reaction times}

In Table 3, mean target reaction times (RT) and mean target error rates for the three distances ( $\mathrm{d} 1, \mathrm{~d} 2$ and $\mathrm{d} 3$ ) of the priming task in block 1 and block 2 are presented. Trials where the numerical value of prime and target were identical (16.7\%) were excluded from the analysis, because it has been shown that repeated presentation of a number can lead to the bypassing of the semantic comparison stage. A repeated measures analysis was conducted on the median reaction times of the participants' correct answers on both prime and target stimulus (on average $4.0 \%$ incorrect trials were removed), with primetarget distance (three levels: distances 1, 2 and 3) and block (two levels: block 1 and block 2 ) as within-subject factors. ${ }^{2}$ The analysis

\footnotetext{
2 Before the analysis on the actual priming distance effect (PDE) was started, an analysis of the performance on the prime stimuli was done as a function of the distance between the prime and the standard 5. This in order to examine the presence of the comparison distance effect $(\mathrm{CDE})$, because it ascertains that the prime stimulus was processed. From the repeated measures analysis, we can conclude that there was a $\mathrm{CDE}$ on reaction times and error rates, indicating that subjects reacted faster and made less errors when distances increased (respectively $F(3,43)=55.79, p<.001$ and $F(3,43)=14.16, p<.001$ ).
} 
Table 3

Mean reaction times (RT), mean error rates and the corresponding (standard deviations) for the three distances (d1-d3) of the priming task in block 1 and block 2 .

\begin{tabular}{lcccc}
\hline & & $\mathrm{d} 1$ & $\mathrm{~d} 2$ & $\mathrm{~d} 3$ \\
\hline RT (ms) & Block 1 & $430.0(72.2)$ & $454.8(78.9)$ & $470.7(75.7)$ \\
& Block 2 & $406.0(65.0)$ & $431.0(69.3)$ & $450.3(82.3)$ \\
Error rates (\%) & Block 1 & $.01(.03)$ & $.05(.07)$ & $.03(.05)$ \\
& Block 2 & $.02(.04)$ & $.04(.06)$ & $.05(.09)$ \\
\hline
\end{tabular}

revealed a main effect of prime-target distance $(F(2,44)=19.26$, $p<.001$ ), indicating that RTs increase when the distances increase (i.e. the PDE): RTs on distances 1,2 and 3 were $418 \mathrm{~ms}, 443 \mathrm{~ms}$ and $460 \mathrm{~ms}$ respectively (see Table 3 ). There was also a main effect of block $(F(1,45)=12.88, p<.01)$ : subjects reacted significantly (on average $23 \mathrm{~ms}$ ) faster in the second block than in the first one. An interaction effect between distance and block was absent $(F<1)$.

\subsubsection{Error rates}

A repeated measures analysis was conducted for the target error rates, using distance (three levels) and block (2 levels) as withinsubject factors. The trials where an incorrect response was given on the prime stimulus (on average $0.1 \%$ ) were excluded from the analysis. A main effect of distance was found $(F(2,44)=8.75, p<.01)$ indicating that subjects made significantly more errors when the distances increase: errors on distances 1,2 and 3 were $1.5 \%, 4.5 \%$ and $4.5 \%$ respectively (see Table 3 ). A main effect of block $(F(1,45)=1.35$, $p=.25)$ and an interaction effect of distance by block $(F<1)$ were absent.

\section{Reliability of the numerical distance effects and correlations between different distance effects}

\subsection{Reliability of the numerical distance effects}

The size of the distance effect was calculated for each participant separately for each block in each task. In order to investigate the reliability of the numerical distance effects, a Pearson correlation was computed between the distance effect observed in the first block and the distance effect in the second block of each task. A significant correlation between the distance effects of both blocks indicates the reliability of the effect (e.g. Borgmann, Risko, Stolz, \& Besner, 2007; Stolz, Besner, \& Carr, 2005). The distance effects in the comparison and the same-different task (CDE and SDDE) were computed by subtracting the average RT (or error rate) on trials with a numerical distance of 3 between both dot patterns from the average RT (error rate) on trials where both magnitudes differed by 1 unit (see also Maloney et al., 2010, for the use of difference scores). The individual distance effect in the priming task was computed slightly different to obtain a positive value of the distance effect. Here, the average RT (or error rate) on trials where prime and target differed by 1 was subtracted from the average RT (error rate) on trials where prime and target differed by 3 . When computing these difference scores, because of the comparability, in all tasks distance 3 was used as the largest numerical distance between two numbers, because this was the largest numerical difference between two congruent stimuli in the priming task. In addition, Cook's values were calculated to detect potential outliers. To interpret Cook's distance, the cutoff values recommended by Bollen and Jackman (1990) were used. In case of the conservative cutoff of 1 for Cook's distance, subjects were immediately excluded from the analyses. In contrast, when subjects reached the sensitive cutoff of $4 / n$ for Cook's distance, it was checked whether the omission of these subjects altered the pattern of results. Providing this was not the case, these subjects were not excluded from the analysis. Based on these criteria, only one subject was removed from the same-different data (see Fig. 1).

For the CDE, a positive significant correlation $(r=.40, p=.01)$ between the $\mathrm{CDE}$ in the first and the second block was found (see Fig. 2). The Pearson correlation between the CDE based on the errors in both blocks was not significant $(r=.22, p=.14)$. With regard to the SDDE a strong positive significant correlation $(r=.65, p<.001)$ between the SDDE in the first block and the second block was observed. The Pearson correlation found between the SDDE based on the errors of the two blocks was also significant $(r=.46 ; p<.01)$. Finally, although visual inspection of Fig. 3 shows a slight tendency to a positive correlation, the Pearson correlation coefficient between the PDE in the two blocks was not significant $(r=.21 ; p=.17$ ). The correlation between the PDE based on the errors also did not reach significance $(r=.11, p=.45)$.

\subsection{Correlations between the different distance effects}

For this analysis, subjects that were outliers in one of the experiments were removed (including the subject that probably counted in experiment 2). The final sample for this analysis consisted of 43 subjects (mean age $=19.44$ years; $S D=6.02 ; 17$ males and 26 females). Pearson correlations were calculated between the mean distance effects observed in the three tasks. The mean distance effect for each task was

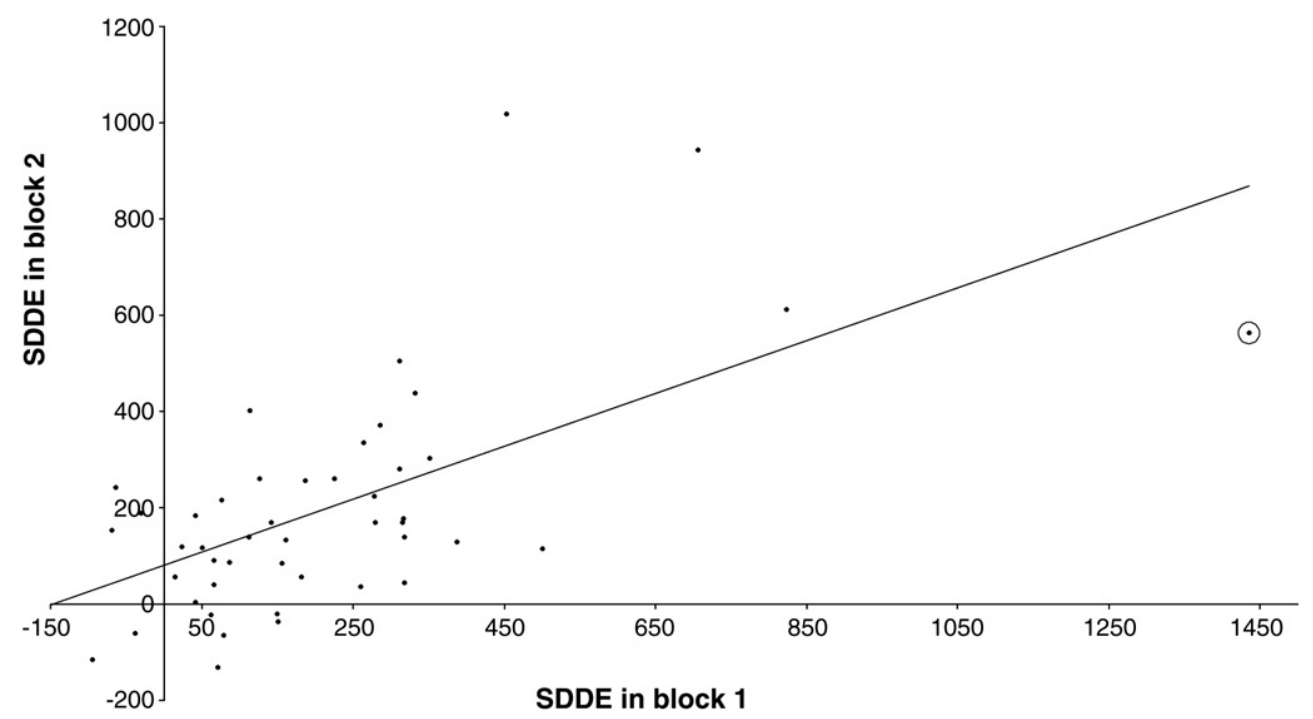

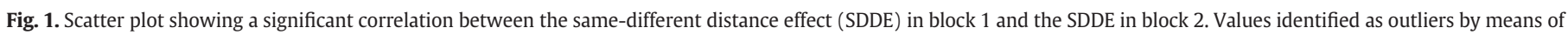
Cook's distance $>1$ are circled. 


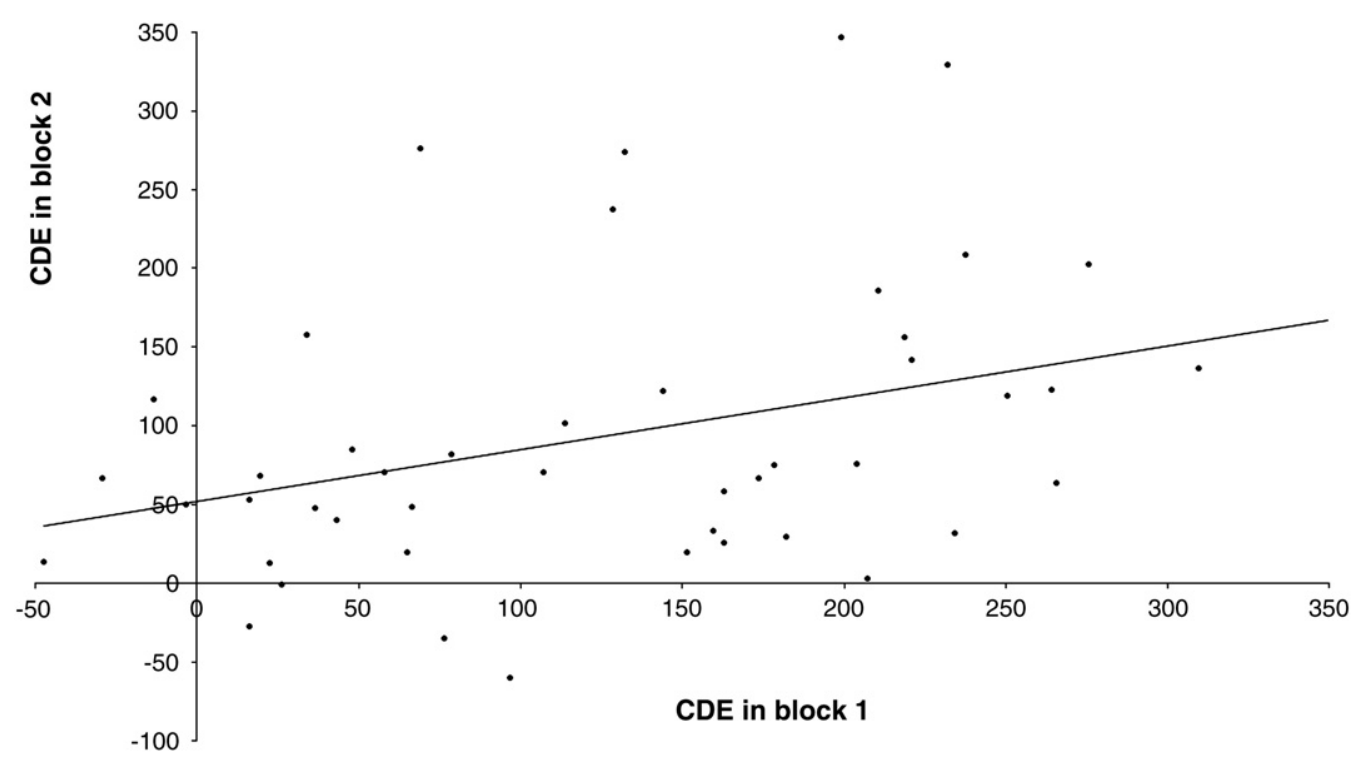

Fig. 2. Scatter plot showing a significant correlation between the comparison distance effect (CDE) in block 1 and the CDE in block 2 .

computed by averaging the distance effects observed in both blocks. This resulted in a strong positive correlation between the CDE and the SDDE $(r=.52, p<.001)$, which indicates that participants with a larger CDE also have a larger SDDE. Between the CDE and the PDE on the one hand and the SDDE and the PDE on the other hand, no significant correlations were found (respectively $r=.03, p=.86$ and $r=.06, p=.68$ ). Between the effects based on the errors, also no significant correlations were found (all $r<.28$, ns).

\section{General discussion}

The development of number processing has been intensively studied examining the performance on basic number tasks (comparison, same-different judgment, and priming). This convincingly showed that latencies and error rates are moderated by the numerical distance between the numbers that need to be processed (i.e. numerical distance effects). The results are usually explained by assuming a magnitude representation related to a mental number line. The reliability of these behavioral measures in mainstream cognitive psychology is typically neglected (see Maloney et al., 2010, for a similar argument). However, the stability of these effects over time, intra-individually, is important, certainly with regard to the developmental literature. Several recent studies have tried to relate the distance effect to general mathematical ability (De Smedt et al., 2009; Holloway \& Ansari, 2009; Iuculano et al., 2008; Mundy \& Gilmore, 2009; Soltész et al., 2010). However, when a behavioral effect is not reliable, the strength of the correlation with other measures like math ability is also limited, or in other words, the observed null result in these cases might reflect a lack of reliability of one of the measures, rather than the absence of a relation between both.

Therefore, a first goal of this study was to examine the test-retest reliability of these effects in each task separately. Again, it should be noted that this study only focused on tasks with non-symbolic stimuli. Research with symbolic stimuli (e.g. Arabic digits) can obviously lead to other results (e.g. Holloway \& Ansari, 2009; Maloney et al., 2010). From our results it can be concluded that in all tasks, the numerical distance effects emerged: participants were faster in comparing two magnitudes that were lying further apart than two close magnitudes (CDE), faster in deciding that two magnitudes that lie further apart were different than two close magnitudes (SDDE) and faster in processing a target number that was preceded by a close prime number than a prime number that lies further apart (PDE). In

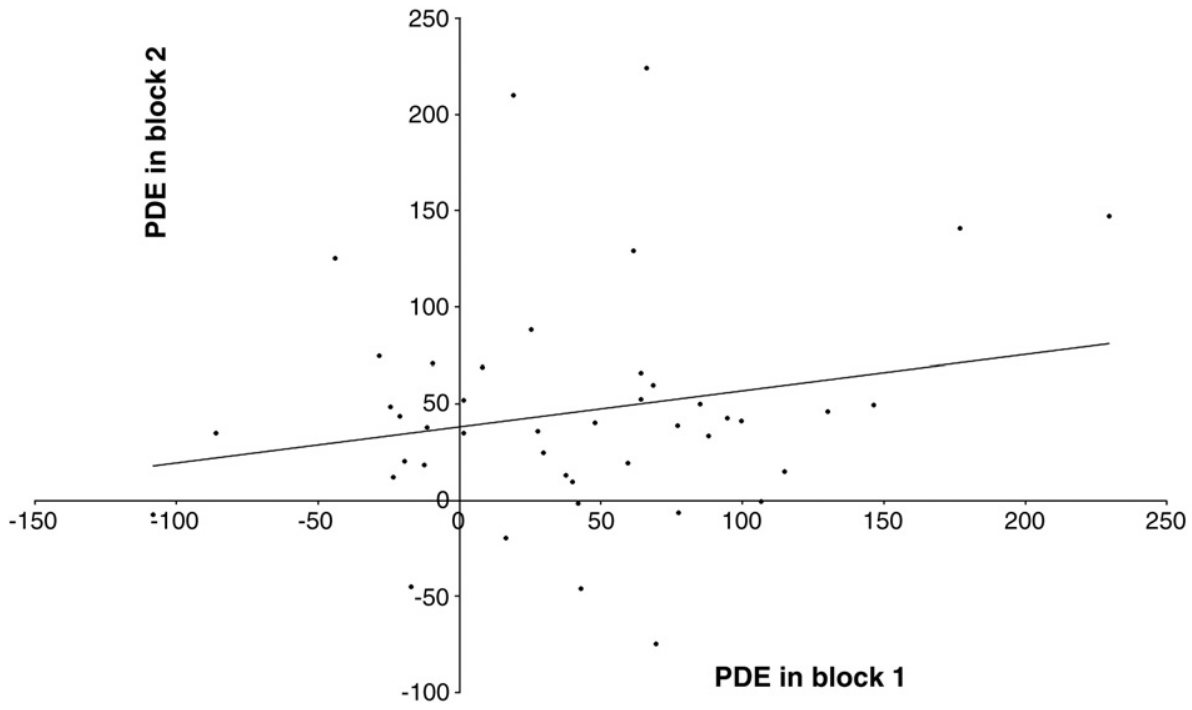

Fig. 3. Scatter plot showing no significant correlation between the priming distance effect (PDE) in block 1 and the PDE in block 2. 
addition, in all tasks, subjects responded significantly faster in the second block than in the first block, suggesting a training-effect. Crucial for the present study purposes, the test-retest reliability of the CDE proved to be significant. In addition, also the SDDE was highly reliable. The reliability of the PDE however, was not significant.

A possible explanation for the unreliability of the PDE could be that the activation of the magnitude representation under automatic conditions is initially vague and needs to be further refined through intentional processing (Cohen-Kadosh \& Walsh, 2009). In contrast, because the CDE and the SDDE rely on intentional processes and thus a more refined representational activation, it is not surprising that these effects are more reliable. This idea is in line with the observations of Stolz et al. (2005) who observed that the reliability of priming effects increased under less automatic conditions.

Furthermore, it should be noted that the reliability coefficients in general are rather low. This may be due to various reasons. One reason is that all distance effects were computed on the basis of difference scores. Several previous studies report an overall decrease in reliability when correlations are calculated using difference scores (e.g. Siegrist, 1995; Strauss, Allen, Jorgensen, \& Cramer, 2005). In a difference score, the measurement errors of both compounds are combined, possibly leading to a reduction of the correlation coefficient (Strauss et al., 2005). Consequently, the reliability of such difference scores would be inherently less reliable than other types of measures. Therefore, in order to examine the reliability of the effects while avoiding the use of difference scores, we additionally computed a Pearson correlation for the distances $\mathrm{d} 1$ and $\mathrm{d} 3$ between the first and the second block in all tasks $^{3}$. All of these correlations were higher than .70 (all $\left.p<.001\right)$ and can be considered as reliable test-retest correlations (Rousson, Gasser, \& Seifert, 2002). These significant correlations show that there is indeed a high test-retest reliability present, but that when using difference scores, these correlations are reduced.

A second reason for the rather low correlation coefficients might be the number of trials that was used. In the case of the $C D E$, for instance, this finding can be considered as a replication of the observations of Maloney et al. (2010) who studied the test-retest reliability of the numerical distance effects observed in different variants of the comparison task. In the non-symbolic variant of the comparison task, these authors found a test-retest correlation of .89 and .84 in experiments 1 and 2 respectively. The difference between these observations and the rather small test-retest correlation of .40 in our first experiment, can be due to the different number of trials that was used. While Maloney et al. (2010) used 40 trials on distances 1 and 4 (the CDE was computed by subtracting the average RT (error rate) of trials with a numerical distance of 4 units between both stimuli from trials where both magnitudes differed by 1 unit), we only used 16 trials on distance 1 and 12 trials on distance 3 in our experiment. Therefore, the smaller number of trials in our study could have induced more noise and consequently have affected the testretest correlation of the $\mathrm{CDE}$ in both blocks. Alternatively, another explanation for the lower reliability of the CDE in the present study in comparison with the Maloney et al. (2010) study might be the fast reaction times. The average reaction times in the present study were about 380 ms faster than in the Maloney et al. (2010) study, possibly due to the instructions which emphasized accuracy, but also, and in particular, speed. These faster reaction times might indicate that subjects relied more on estimation than on counting strategies in the present study, resulting in less precise representations of magnitudes, and leading in this way to a less reliable CDE.

A second goal of this study was to investigate the relation between the numerical distance effects observed in non-symbolic comparison,

\footnotetext{
${ }^{3}$ In the case of the comparison task, the correlations were .71 and .72 for distances 1 and 3 respectively (all $p<.001$ ). In the same-different task, the correlations were .82 and .70 for distances 1 and 3 respectively (all $p<.001$ ). Finally, in the priming tasks, these correlations were .73 and .71 respectively (all $p<.001$ )
}

same-different judgment and priming within the same sample of subjects. Several authors (e.g. Cohen-Kadosh \& Walsh, 2009; Verguts et al., 2005) have already suggested that the numerical distance effects may be different in the different tasks. Based on computational modeling, Verguts and colleagues (e.g. Van Opstal \& Verguts, 2010; Verguts et al., 2005) proposed that the numerical distance effects can differ due to different underlying mechanisms. On the basis of a literature review of behavioral and imaging studies, Cohen-Kadosh and Walsh (2009) suggested on the other hand that differences between the distance effects in the diverse tasks can be explained by differences between automatic and intentional processing. We found a correlation between the CDE and the SDDE, which can be considered as a confirmation of the results of Piazza, Izard, Pinel, Le Bihan, and Dehaene (2004), who observed similar discrimination performances in a same-different task and a comparison task. In line with CohenKadosh and Walsh (2009), this can be explained by the fact that both the CDE and the SDDE are 'intentional' effects, because in both tasks, the numerical value of both stimuli is essential for the task requirements. Moreover, although this finding is at first sight not in line with the computational model of Verguts et al. (2005), representational overlap remains a candidate to explain the correlation between the SDDE and the CDE. As mentioned in Verguts et al. (2005), representational overlap does not seem to be a necessary condition for a $\mathrm{CDE}$ to emerge, but it still does play a role.

The absence of the correlation with the PDE, however, can be explained by the lack of reliability of this measure.

\section{Conclusion}

In this study, two issues were addressed: the reliability of the different non-symbolic numerical distance effects (i.e. comparison distance effect, same-different distance effect and priming distance effect), and the relation between these effects. First, the test-retest analyses showed that the comparison distance effect (CDE) and the same-different distance effect (SDDE) are indeed reliable measures of how people process numbers. The priming distance effect (PDE) was not reliable, certainly when using difference scores to compute this effect. Consequently, some caution is warranted concerning the use of the PDE: although this has proven to be a relatively robust measure on the group level (Reynvoet et al., 2009; Stolz et al., 2005), the size of the PDE is variable within the same subjects measured on different occasions. Second, when investigating the relation between the different effects, we found a correlation between the CDE and the SDDE. In sum, it can be concluded that the numerical distance effects observed in comparison and same-different tasks are trustworthy measures to use in future (developmental) research, for instance when investigating the relation between these measures and general math ability, whereas caution is required regarding the reliability for the numerical distance effect observed in priming tasks.

\section{Acknowledgments}

This research was supported by the Research Fund K.U. Leuven. DS, ED and BR are affiliated to the Laboratory of Experimental Psychology of the Department of Psychology, University of Leuven and to the Subfaculty of Psychology and Educational Sciences, University of Leuven - Campus Kortrijk.

\section{References}

Ansari, D. (2008). Effects of development and enculturation on number representation in the brain. Nature Reviews. Neuroscience, 9, 278-291.

Bollen, K. A., \& Jackman, R. (1990). Regression diagnostics: An expository treatment of outliers and influential cases. In J. Fox, \& J. Scott Long (Eds.), Modern methods of data analysis (pp. 257-291). Newbury Park, CA: Sage.

Borgmann, K. W. U., Risko, E. F., Stolz, J. A., \& Besner, D. (2007). Simon says: Reliability and the role of working memory and attentional control in the Simon Task. Psychonomic Bulletin \& Review, 14, 313-319. 
Buckley, P. B., \& Gillman, C. B. (1974). Comparisons of digits and dot patterns. Journal of Experimental Psychology, 103, 1131-1136.

Cohen, D. J. (2009). Integers do not automatically activate their quantity representation. Psychonomic Bulletin \& Review, 16, 332-336.

Cohen-Kadosh, R. (2008). Numerical representation: Abstract or non-abstract? The Quarterly Journal of Experimental Psychology, 61, 1160-1168.

Cohen-Kadosh, R. C., Lammertyn, J., \& Izard, V. (2008). Are numbers special? An overview of chronometric, neuroimaging, developmental, and comparative studies of magnitude representation. Progress in Neurobiology, 84, 132-147.

Cohen-Kadosh, R., \& Walsh, V. (2009). Numerical representation in the parietal lobes: Abstract or not abstract? The Behavioral and Brain Sciences, 32, 313-328.

Dehaene, S. (1997). The number sense: How the mind creates mathematics. New York: Oxford University press.

Dehaene, S., \& Akhavein, R. (1995). Attention, automaticity, and levels of representation in number processing. Journal of Experimental Psychology. Learning, Memory, and Cognition, 21, 314-326.

Dehaene, S., Bossini, S., \& Giraux, P. (1993). The mental respresentation of parity and number magnitude. Journal of Experimental Psychology: General, 122, 371-396.

Dehaene, S., \& Changeux, J. P. (1993). Development of numerical abilities: A neuronal model. Journal of Cognitive Neuroscience, 5, 390-407.

Dehaene, S., Izard, V., \& Piazza, M. (2005). Control over non-numerical parameters in numerosity experiments. Unpublished manuscript (available on www.unicog.org).

Dehaene, S., Piazza, M., Pinel, P., \& Cohen, L. (2003). Three parietal circuits for number processing. Cognitive Neuropsychology, 20, 487-506.

De Smedt, B., Verschaffel, L., \& Ghesquière, P. (2009). The predictive value of numerical magnitude comparison for individual differences in mathematics achievement Journal of Experimental Child Psychology, 103, 469-479.

Duncan, E. M., \& McFarland, C. E. (1980). Isolating the effects of symbolic distance and semantic congruity in comparative judgments: An additive-factors analysis. Memory and Cognition, 8, 612-622.

Herrera, A., \& Macizo, P. (2008). Cross-notational semantic priming between symbolic and non-symbolic numerosity. The Quarterly Journal of Experimental Psychology, 61, $1538-1552$.

Holloway, I. D., \& Ansari, D. (2008). Domain-specific and domain-general changes in children's development of number comparison. Developmental Science, 11, 644-649.

Holloway, I. D., \& Ansari, D. (2009). Mapping numerical magnitudes onto symbols: The numerical distance effect and individual differences in children's mathematics achievement. Journal of Experimental Child Psychology, 103, 17-29.

Iuculano, T., Tang, J., Hall, C., \& Butterworth, B. (2008). Core information processing deficits in developmental dyscalculia and low numeracy. Developmental Science, 11, 669-680.

Koechlin, E., Naccache, L., Block, E., \& Dehaene, S. (1999). Primed numbers: Exploring the modularity of numerical representations with masked and unmasked semantic priming. Journal of Experimental Psychology: Human Perception and Performance, 25, 1882-1905.

Maloney, E., Risko, E. F., Preston, F., Ansari, D., \& Fugelsang, J. (2010). Challenging the reliability and validity of cognitive measures: The case of the numerical distance effect. Acta Psychologica, 134, 154-161.
Moyer, R. S., \& Landauer, T. K. (1967). Time required for judgments of numerical inequality. Nature, 215, 1519-1520.

Mundy, E., \& Gilmore, C. K. (2009). Children's mapping between symbolic and nonsymbolic representations of number. Journal of Experimental Child Psychology, 103, 490-502.

Noel, M. -P., Rouselle, L., \& Mussolin, C. (2005). Magnitude representation in children. Its development and dysfunction. In J. Campbell (Ed.), Handbook of mathematical cognition (pp. 179-197). New York, NY: Psychology Press.

Piazza, M., Izard, V., Pinel, P., Le Bihan, D., \& Dehaene, S. (2004). Tuning curves for approximate numerosity in the human intraparietal sulcus. Neuron, 44, 547-555

Restle, F. (1970). Speed of adding and comparing numbers. Journal of Experimental Psychology, 91, 191-205.

Reynvoet, B., Brysbaert, M., \& Fias, W. (2002). Semantic priming in number naming. Quarterly journal of Experimntal Psychology, 55A, 1127-1139.

Reynvoet, B., Caessens, B., \& Brysbaert, M. (2002). Automatic stimulus-response associations may be semantically mediated. Psychonomic Bulletin \& Review, 9, $107-112$.

Reynvoet, B., De Smedt, B., \& Van den Bussche, E. (2009). Children's representation of symbolic magnitude: The development of the priming distance effect. Journal of Experimental Child Psychology, 103, 480-489.

Reynvoet, B., \& Notebaert, K. (2009). Abstract or not? Insights from priming. The Behavioral and Brain Sciences, 32, 349-350.

Rousson, V., Gasser, T., \& Seifert, B. (2002). Assessing intrarater, interrater and test-retest reliability of continuous measurements. Statistics in Medicine, 21, 3431-3446.

Siegrist, M. (1995). Reliability of the Stroop test with single-stimulus presentation. Perceptual and Motor Skills, 81, 1295-1298.

Sekuler, R., \& Mierkiewicz, D. (1977). Children's judgments of numerical inequality Child Development, 48, 630-633.

Soltész, F., Szücs, D., \& Szücs, L. (2010). Relationships between magnitude representation, counting and memory in 4- to 7-year-old children: A developmental study. Behavioural and Brain Functions, 6, 13. doi:10.1186/1744-9081-6-13.

Stolz, J. A., Besner, D., \& Carr, T. H. (2005). Implications of measures of reliability for theories of priming: Activity in semantic memory is inherently noisy and uncoordinated. Visual Cognition, 12, 284-336.

Strauss, G. P., Allen, D. N., Jorgensen, M. L., \& Cramer, S. L. (2005). Test-retest reliability of standard and emotional Stroop Tasks. An investigation of color-word and picture-word versions. Assessment, 12, 330-337.

Van Opstal, F., Gevers, W., De Moor, W., \& Verguts, T. (2008). Dissecting the symbolic distance effect: Priming and comparison distance effects in numerical and nonnumerical orders. Psychonomic Bulletin E Review, 15, 419-425.

Van Opstal, F., \& Verguts, T. (2010). The origins of the numerical distance effect: The same different task, European Journal of Cognitive Psychology, First published on: 22 July 2010 (iFirst). doi:10.1080/09541441003662742.

Verguts, T., Fias, W., \& Stevens, M. (2005). A model of exact small-number representation. Psychonomic Bulletin \& Review, 12, 66-80. 